\title{
ANÁLISIS DE LOS PATRONES ACÚSTICOSE INTERVENCIÓN LOGOPÉDICA MEDIANTE LA TÉCNICA LAX VOX EN LA DISTROFIA MUSCULAR OCULOFARÍNGEA. DESCRIPCIÓN DE UN CASO
}

\section{Analysis of acoustic patterns and logopedic intervention using Lax Vox technique in oculopharyngeal dystrophy. Description of a case}

\author{
Tatiana ROMERO-ARIAS ${ }^{1 ; 2}$; Cristina HERNÁNDEZ-KAUFFMAN ${ }^{3}$; Moisés BETANCORT-MONTESINOS ${ }^{4}$; \\ María Teresa TORRES-LARROSA ${ }^{5}$ \\ ${ }^{1}$ Departamento de Psicología Cognitiva, Social y Organizacional. Universidad de La Laguna. Tenerife. Islas \\ Canarias. España. \\ ${ }^{2}$ Instituto Universitario de Neurociencia (IUNE). Universidad de La Laguna. Tenerife. Islas Canarias. España. \\ ${ }^{3}$ Servicio de Logopedia. Hospital Universitario Nuestra Señora de Candelaria (HUNSC). Tenerife. Islas Canarias. \\ España. \\ ${ }^{4}$ Departamento de Psicología Clínica, Psicobiología y Metodología. Universidad de La Laguna. Tenerife. Islas \\ Canarias. España. \\ ${ }^{5}$ Servicio de Otorrinolaringología. Hospital Universitario Nuestra Señora de Candelaria (HUNSC). Tenerife. \\ Islas Canarias. España.
}

Correspondencia: tromeroa@ull.edu.es

Fecha de recepción: 16 de octubre de 2018

Fecha de aceptación: 14 de noviembre de 2018

Fecha de publicación: 16 de noviembre de 2018

Fecha de publicación del fascículo: 1 de junio de 2020

Conflicto de intereses: Los autores declaran no tener conflictos de intereses

Imágenes: Los autores declaran haber obtenido las imágenes con el permiso de los pacientes

Política de derechos y autoarchivo: se permite el autoarchivo de la versión post-print (SHERPA/RoMEO)

Licencia CC BY-NC-ND. Licencia Creative Commons Atribución-NoComercial-SinDerivar 4.0 Internacional

Universidad de Salamanca. Su comercialización está sujeta al permiso del editor

RESUMEN: Introducción y objetivo: La distrofia muscular oculofaríngea es una enfermedad genética que afecta a los músculos proximales de las extremidades, ptosis de los párpados y disfagia orofaríngea. En consecuencia, el perfil clínico de estos pacientes podría estar acompañado de disfonía, disartria y pérdida 
de peso. Las alteraciones de la voz, la articulación y la deglución caracterizan el perfil de la disartrofonía. Se presenta un caso clínico de una paciente con distrofia muscular oculofaríngea que ha sido remitida al servicio de logopedia con el objetivo de rehabilitar su voz. Descripción: La novedad que presenta este caso en la intervención logopédica, es que ha sido rehabilitada mediante técnicas de tracto vocal semiocluído. Resultados: Los resultados obtenidos muestran la efectividad del Lax vox, en la rehabilitación de la paciente. Discusión: En la búsqueda bibliográfica hemos encontrado evidencia científica de la efectividad del Lax Vox en pacientes con disfonía, nódulos, pólipos, edemas; pero no hemos encontrado evidencia de la efectividad del Lax Vox en pacientes con distrofia muscular oculofaríngea. Conclusiones: El uso de la técnica Lax vox para la rehabilitación de los pacientes con distrofia muscular óculo-faríngea es beneficiosa.

PALABRAS CLAVE: patrones acústicos; voz patológica; rehabilitación logopédica; distrofia muscular oculofaríngea; tracto vocal semiocluído.

SUMMARY: Introduction and objective: Oculopharyngeal muscular dystrophy is a genetic disease that affects the proximal muscles of the extremities, ptosis of the eyelids and oropharyngeal dysphagia. Consequently, the clinical profile of these patients could be accompanied by dysphonia, dysarthria and weight loss. The alterations of the voice, the articulation and the swallowing characterize the profile of the disartrofonía. We present a clinical case of a patient with oculopharyngeal muscular dystrophy who has been referred to the sevice of speech therapy with the aim of rehabilitating her voice. Description: The novelty that this case presents in the speech therapy intervention is that it has been rehabilitated using semiocluid vocal tract techniques. Results: The results obtained show the effectiveness of Lax vox in the rehabilitation of the patient. Discussion: In the literature search we have found scientific evidence of the effectiveness of Lax Vox in patients with dysphonia, nodules, polyps, edema; but we have not found evidence of the effectiveness of Lax Vox in patients with oculopharyngeal muscular dystrophy. Conclusions: With these results, we can conclude that the use of the Lax vox technique for the rehabilitation of patients with oculopharyngeal muscular dystrophy is beneficial.

KEYWORDS: acoustic patterns; pathological voice; logopedic rehabilitation; oculopharyngeal muscular dystrophy; Semi-fluid vocal tract.

\section{INTRODUCCIÓN}

La distrofia muscular oculofaríngea (DMOF) es una enfermedad genética con un patrón predominantemente autosómico dominante, relacionado con el gen PABPN1 [1]. La DMOF fue descrita por primera vez por Taylor, en 1915, como una miopatía hereditaria de transmisión autosómica dominante de manifestaciones tardías (entre la quinta y la sexta década de la vida), con signos clínicos de debilidad de los músculos proximales de las extremidades, ptosis de los párpados y disfagia orofaríngea, esta última posiblemente asociada a debilidad muscular proximal [2-4]. Los pacientes que presentan DMOF son remitidos directamente al digestivo desde su médico de familia y no con tanta frecuencia al otorrinolaringólogo, por lo que el conocimiento de esta enfermedad es fundamental [5]. La DMOF se caracteriza por disfagia en diferentes niveles, de acuerdo con la gravedad o la progresión del trastorno, siendo necesario modificar la rigidez de la dieta. Los desórdenes de la deglución son, por lo tanto, progresivos, comenzando por digerir la ingesta de alimentos sólidos, lo que lleva a una dieta más suave y, posteriormente, suprime la ingesta de alimentos por vía oral. En consecuencia, es probable que estos pacientes presenten episodios de broncoaspiración, regurgitación oral y / o reflujo nasal, crisis de asfixia, neumonía y asma bronquial 
[3]. El perfil clínico podría estar acompañado de disfonía [3], disartria [6] y pérdida de peso [3]. Las alteraciones de la voz, la articulación y la deglución caracterizan el perfil de la disartrofonía, lo que repercute negativamente en la calidad de vida de estos pacientes [7]. El digestivo y el nutricionista se encargan del tratamiento para la disfagia, pero y ¿qué pasa con la voz?

En este artículo presentamos el caso clínico de una paciente con DMOF que después de seguir el tratamiento para la disfagia es remitida a los servicios de otorrinolaringología y logopedia para llevar a cabo una intervención/rehabilitación logopédica.

\section{DESCRIPCIÓN}

Se presenta el caso clínico de una mujer de 70 años diagnosticada de DMOF. Es la tercera persona de su familia en padecer esta enfermedad. Sus hijos hasta la fecha no han manifestado ningún síntoma. La paciente es exfumadora social desde hace 25 años. Durante la noche presenta un ronquido leve sin otros síntomas de SAHS o hipoventilación. Duerme con cierta inclinación. La paciente tiene sensación de goteo nasal posterior con expulsión de secreciones blanquecinas ocasionalmente verdosas. Disnea en cuestas. Después de ser tratada por su disfagia, es remitida al servicio de otorrinolaringología y al servicio de logopedia para hacer rehabilitación logopédica con el fin de mejorar la calidad de su voz. En el examen de nasofibroscopia, la paciente presenta cuerdas vocales de aspecto y motilidad normal (Figura 1). Al hacerle tragar agua, después de la deglución queda algo de agua acumulada en los senos piriformes. Presenta una voz nasal con escapes de aire y carece de coordinación fonorrespiratoria por lo que su intensidad es muy baja. Con este cuadro clínico, el servicio de logopedia ha diseñado un plan de 10 sesiones con la finalidad de mejorar su calidad de voz.

Durante las 10 sesiones de rehabilitación logopédica, los objetivos de la intervención logopédica son los siguientes: rehabilitar la disartrofonía, eliminar en la medida de lo posible la voz nasal, reducir el F0 y F1 utilizando la técnica TVSO Lax Vox e instaurar las pautas de higiene vocal para romper el círculo del sobreesfuerzo vocal. Para ello utilizamos como instrumentos: nasofibroscopio, software Praat, Scape-scope, Lax Vox. Tubo de silicona de $35 \mathrm{~cm}$ de largo. Diámetro externo de 12 $\mathrm{mm}$, interno de $8 \mathrm{~mm}$. Botella con $4-5 \mathrm{~cm}$ de agua.

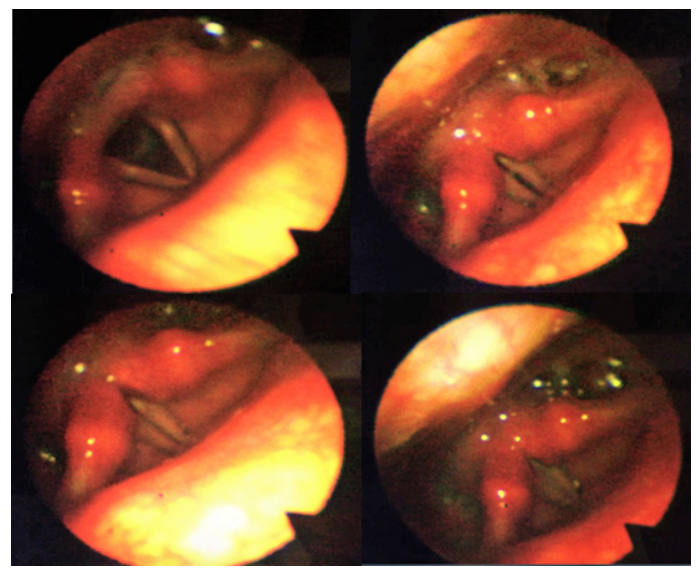

Figura 1. Imágenes de nasofibroscopia de la paciente.

\section{DISCUSIÓN}

Antes de empezar la intervención logopédica se recogen datos acústicos de la paciente con el software Praat (Figura 2 A y B). Una vez analizados estos resultados, se elaboró un plan de intervención ajustado a las necesidades de la paciente utilizando la técnica de tracto vocal semiocluído (TVSO), Lax vox. La mayoría de los programas de rehabilitación fisiológica, se basan en ejercicios de TVSO. Este tipo de ejercicios hacen referencia a una serie de posturas cuya finalidad es alargar u ocluir parcialmente el tracto vocal [8], provocando un cambio en el patrón vibratorio de los pliegues vocales. Los ejercicios que se incluyen bajo la denominación de TVSO son: vibración de labios, vibración de lengua, humming, fricativos bilabiales y fonación con tubos de diferentes diámetros [9]. Los tubos de 
resonancia pueden ser utilizados de dos maneras: dejando el extremo distal del tubo libre en el aire y la otra forma es sumergiendo el extremo distal en agua: en ambos casos el tubo se mantiene derecho en la boca del participante, actuando como una extensión artificial del tracto vocal [10]. Dentro de la fonación con tubos de diámetros diferentes, se encuentra el Lax Vox. El Lax Vox es una técnica que se utiliza para el tratamiento de la voz con la que se pretende alargar el tracto vocal de forma artificial semiocluyéndolo y de esta forma generar atenuación en la impedancia glótica y aumento de la impedancia de dicho tracto vocal, creando un TV en forma de megáfono invertido [11]. De esta manera se genera un cambio positivo en la vibración de las cuerdas vocales. Esta técnica se emplea durante el tratamiento de patologías de la voz con fatiga vocal, tensión en la musculatura laríngea, nódulos, pólipos, edemas y también en voces normales para el entrenamiento y calentamiento de la voz. En el ámbito terapéutico, los tubos de resonancia han sido aplicados en diferentes casos de patología vocal, incluyendo disfonía funcional y pacientes con nódulos [12,13]; mientras que no hay evidencia científica en casos de DMOF. Otras investigaciones de fonación con tubos encontraron un aumento de la impedancia de entrada en el rango de frecuencia fundamental por el descenso de la frecuencia del primer formante [14]. Sampaio, Oliveria y Behlau en 2008, estudiaron los efectos inmediatos de ejercicios de TVSO (finger kazoo y fonación con tubos) y encontraron resultados positivos en la autovaloración vocal y valoración acústica, en los cuales había una disminución de F0, tanto con finger kazoo como con ejercicios de fonación con tubo. También encontraron resultados positivos en la valoración perceptivo-auditiva sólo en la fonación con tubos [11]. Desde el punto de vista acústico uno de los beneficios de la fonación con tubos y oclusiones parciales del tracto vocal es el descenso del primer formante (F1), por lo tanto, la F0 puede ser producida cerca de F1; lo que permitiría experimentar efectos de un bajo umbral de presión de la fonación, una disminución del flujo de aire transglótico y una voz rica en armónicos [10]. Hay pocas investigaciones de intervención logopédica con ejercicios de TVSO, pero todas encuentran cambios positivos en la calidad de la voz de los participantes. Una vez finalizadas las 10 sesiones, se recogen muestras acústicas de la paciente (Figura 2 B y C) y se comparan con las iniciales.

En cuanto a los análisis acústicos, encontramos diferencias en los formantes de la voz (Tabla 1). En las tablas podemos apreciar cómo todos los formantes disminuyen sus $\mathrm{Hz}$. Estos resultados son convergentes con los obtenidos por con los de Story, Laukkanen y Titze en el año 2000; y con los resultados de Sampaio, Oliveria y Behlau en 2008. Estos autores encuentran un descenso inmediato de los formantes utilizando la fonación con tubos en la rehabilitación de la voz de sus pacientes; consiguiendo así rehabilitar las patologías y mejorar la calidad de la voz de sus pacientes.

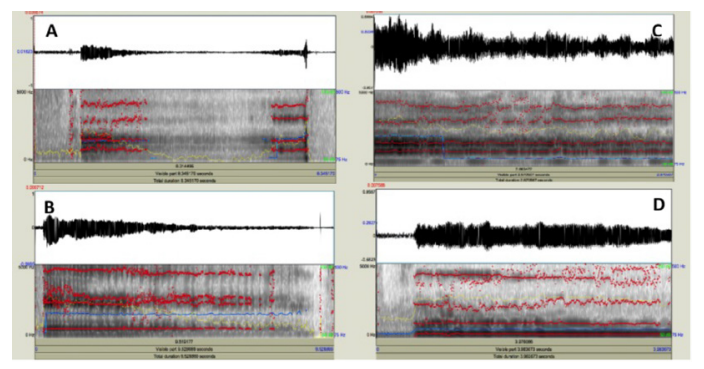

Figura 2. Grabaciones con el software Praat. A y B son los audios preintervención logopédica. C y D son los audios posintervención logopédica.

Por otro lado, encontramos diferencias favorables en la intensidad de fonación. En el registro de voz previo a la intervención, la paciente tenía una intensidad de $62,46 \mathrm{~dB}$; mientras que en el registro de voz posterior a la intervención su intensidad fue de $75,79 \mathrm{~dB}$. Estos resultados son favorables ya que la paciente presentaba una mala coordinación fonorrespiratoria y había escape de aire nasal, por lo que su intensidad era muy baja. El escape 
de aire nasal lo entrenamos con el Scape-scope. En las primeras sesiones la paciente expulsaba gran cantidad de aire por ambas narinas, pero con el entrenamiento consiguió dejar de expulsar aire nasal. Desde que consiguió controlar el escape de aire nasal, su voz cambió por completo. Actualmente, nos encontramos con una voz con una intensidad aceptable y respecto al inicio, ha perdido nasalidad. Los ejercicios de articulación también han servido de apoyo para trabajar todo lo anterior. A nivel fisiológico, en la nasofibroscopia encontramos que las cuerdas continúan teniendo una motilidad normal y que el aparato fonador se encuentra en buen estado.

\section{CONCLUSIONES}

Con estos resultados podemos concluir que el uso de la técnica Lax vox para la rehabilitación de los pacientes con distrofia muscular oculofaríngea es beneficiosa.

Tabla 1. Se presentan las puntuaciones en $\mathrm{Hz}$ de los formantes de la voz antes de la intervención logopédica y posterior a la intervención.

\begin{tabular}{|l|c|c|c|c|c|}
\hline & F0 Hz & F1 Hz & F2 Hz & F3 Hz & F4 Hz \\
\hline Preintervención & 839.7 & 932.94 & 1833.73 & 2904.57 & 394.72 \\
\hline Posintervención & 727.9 & 230.76 & 848.82 & 1574.95 & 3233.56 \\
\hline
\end{tabular}

\section{BIBLIOGRAFÍA}

1. Maeda M, Hashimoto T, Oliveira I, Rodrigues L. Oculopharyngeal muscular dystrophy or oculopharyngeal distal myopathy: case report. Braz J Otorhinolaryngol. 2017;83(2):235-8.

2. Tremolizzo L, Galbussera A, Tagliabue E, Fermi S, Bruttini M, Lamperti C, et al. An apparently sporadic case of oculopha- ryngeal muscular dystrophy: the first Italian report. Neurol Sci. 2007;28:339-41.

3. Medici M, Pizzarossa C, Skuk D, Yorio D, Emmanuelli G, Mesa R. Oculopharyngeal muscular dystrophy in Uruguay. Neuromuscular Disorders. 1997;7(Suppl.1):50-2.
4. Liaño AD, Fernandez R, Yárnoz C, Artieda C, González G, Artajona A et al. Distro a muscular oculofaríngea. Tratamiento quirúrgico. Rev. Chilena de Cirugía. 2009;61(4):360-5.

5. Hernández E, Mesa M, de Frías B, Rivas P. Oculopharyngeal Muscular Dystrophy: A case report and review of the literatura. Acta Otorrinolaringol Esp.2012;63(6):482-4.

6. Morales SG, Méndez LA, Rivero AR, Sánchez VML. Distrofia muscular progresiva. Rev Hosp Jua Mex 2002;69(1): 30-7.

7. Barata FL, Miguel LS, Silva SAC, Carrara-de Angelis E. Caracterização da fonoarticulação e sua relação com a disfagia em pacientes com disartrofonia em um hospital oncológico. Distúrb Comum. 2009;21(1):79-91.

8. Nix J, Simpson C. Semi-occluded vocal tract postures and their applications in the singing voice studio. J Singing. 2008;64(3):339-42.

9. Cecconello L. Ejercicios de tracto vocal semiocluido, XII Jornadas Foniátricas, Universidad Nacional de San Luis, Facultad de Ciencias Humanas, San Luis, Argentina, 2009, CD ROM, ISBN 978-987-1595-02-0.

10. Guzmán M, Higueras D, Fincheira C, Muñoz D, Guajardo C. Immediate effects of a vocal exercise sequence with resonance tubes. CEFAC. 2012;14(3):471-80.

11. Sampaio M, Oliveira G, Behlau M. Investigação de efeitos imediatos de dois exercícios de tracto vocal semi-ocluído.Pro-Fono Revista de Atualização Científica.2008;20(4):261-6.

12. Simberg S. Prevalence of vocal symptoms and voice disorders among teacher students and teachers and a model of early interevention. Finland:University of Helsinki, Departament of Speech Sciences;2004.

13. Simberg $\mathrm{S}$. The resonante tuve-a versatile device in voice therapy. Kjaer BE(ed):Nine paper on Logopedics and Phoniatrics fron 5th Nordic Congress of Logopedics anda Phoniatrics, Helsinki. 2000;81-5.

14. Story B, Laukkanen A, Titze I. Acoustic Impedance of an Artificially Lengthened and Constricted Vocal Tract. J.Voice. 2000;14(4):455-69 